\section{Rare Case of Septic Arthritis Caused by Candida krusei: Case Report and Literature Review}

\section{To the Editor:}

Acute monoarthritis is a common rheumatological emergency that requires immediate investigation to rule out a possible infection. Candida species can be rarely isolated from joint infections ${ }^{1}$; however, as more aggressive strategies are used to treat patients with hematological malignancies, new pathogens have emerged ${ }^{2}$.

We describe a 75-year-old white man with a diagnosis of acute myeloid leukemia (AML) who was admitted to the University of Texas M.D. Anderson Cancer Center with neutropenic fever and sudden onset of severe right knee pain without trauma. His history was significant for diabetes mellitus and relapsing AML refractory to treatment. At the time of admission he had been neutropenic for a year and was on his first course (day 47) of salvage therapy with fludarabine and cytarabine. He was receiving prophylaxis with fluconazole $200 \mathrm{mg} /$ daily and valacyclovir $500 \mathrm{mg}$ /daily for the past 4 months and levofloxacin $500 \mathrm{mg} /$ daily for the past year. Antifungal prophylaxis was switched to voriconazole $200 \mathrm{mg}$ twice daily, 1 month before admission, around the same time he developed right lower extremity cellulitis, treated with broad-spectrum antibiotics.

On examination, he was febrile at $38.3^{\circ} \mathrm{C}$ and his right knee was swollen and tender with no erythema. Passive and active range of motion was painful and limited. Initial investigations revealed serum white blood cell count $200 / \mu 1$ with an absolute neutrophil count $90 / \mu 1$. Chest radiograph was normal and plain radiographs of the knees showed chondrocalcinosis. Differential diagnosis included septic versus crystal arthritis. Arthro-centesis of the knee yielded $60 \mathrm{cc}$ of clear fluid that contained 5-10 white blood cells/high power field with no crystals. Staining of synovial fluid demonstrated no bacterial, fungal, or mycobacterial infection. The patient was then empirically treated with intravenous meropenen and daptomycin. Blood and urine cultures at admission were negative. By the fifth day, culture of the synovial fluid was positive for a yeast identified as Candida kru$s e i$. He was initially treated with posaconazole $400 \mathrm{mg}$ twice daily orally for 10 days. He was then switched to caspofungin intravenously when symptoms did not improve. While on caspofungin he developed a bacterial bronchopneumonia and died from septic shock 21 days after starting on caspofungin.
Fungal arthritis caused by Candida species is uncommon. Although infrequent, $C$. albicans is the species most frequently isolated from a fungal-infected joint ${ }^{1}$. According to previous reports, the knees and intervertebral disks are affected more frequently. Fungal arthritis is most often due to hematogenous seeding rather than to direct inoculation of fungi, and it occurs in patients with predisposing factors ${ }^{1}$

Cancer patients have a higher incidence of fungemia than do noncancer patients, with leukemia patients accounting for $25 \%$ of reported cases ${ }^{3}$. Neutrophils are critical for protection against systemic infections and neutropenic patients have a much higher rate of visceral dissemination and death ${ }^{2,3}$. Since the introduction of fluconazole for fungal prophylaxis in neutropenic patients, the incidence of $C$. albicans fungemia has decreased dramatically, but has shifted toward a greater involvement of non-albicans infections over the last 2 decades $^{2}$.

C. krusei is highly vulnerable to enzymes in granules of neutrophils, thus it shows very low pathogenicity and invasiveness ${ }^{4}$. Its main virulence is due to a multilayered hydrophobic cell wall, which makes it easier for this fungus to adhere to and colonize inert surfaces and to develop an extensive biofilm on catheter disks ${ }^{4}$. Nearly $90 \%$ of the documented infections due to $C$. krusei are fungemia ${ }^{4}$. The most remarkable feature of $C$. krusei is its intrinsic resistance to fluconazole ${ }^{2}$. It remains susceptible to voriconazole, posaconazole, ravubconazole, and caspofungin. Indeed, fluconazole prophylaxis along with neutropenia and bone marrow transplant are considered specific risk factors associated with C. krusei $i^{5}$.

In patients with high risk of invasive fungal infection, prophylaxis with voriconazole $200 \mathrm{mg}$ twice daily has been shown to be as effective as prophylaxis with fluconazole $400 \mathrm{mg}$ /daily in achieving fungal-free survival ${ }^{2}$.

Our patient represents the sixth case of fungal arthritis caused by $C$. kruse $i$ that has been reported in the last 30 years. These include a case of a heroin addict ${ }^{6}$ and 5 cases of patients with hematologic malignancies $7,8,9,10$ (Table 1). All 5 patients with hematologic malignancies were neutropenic and 3 of them had been receiving fluconazole at the time of the diagnosis of fungal arthritis. The $C$. krusei isolated from our patient was sensitive to voriconazole in vitro. This case may represent a failure of prophylaxis, which cautions against the false sense of assurance when the patient is receiving appropriate prophylaxis in a neutropenic state. However, our patient developed cellulitis of the same limb at the time of the switch of fluconazole to voriconazole. The cellulitis on the same limb may have served

Table 1. Reported cases of Candida krusei arthritis.

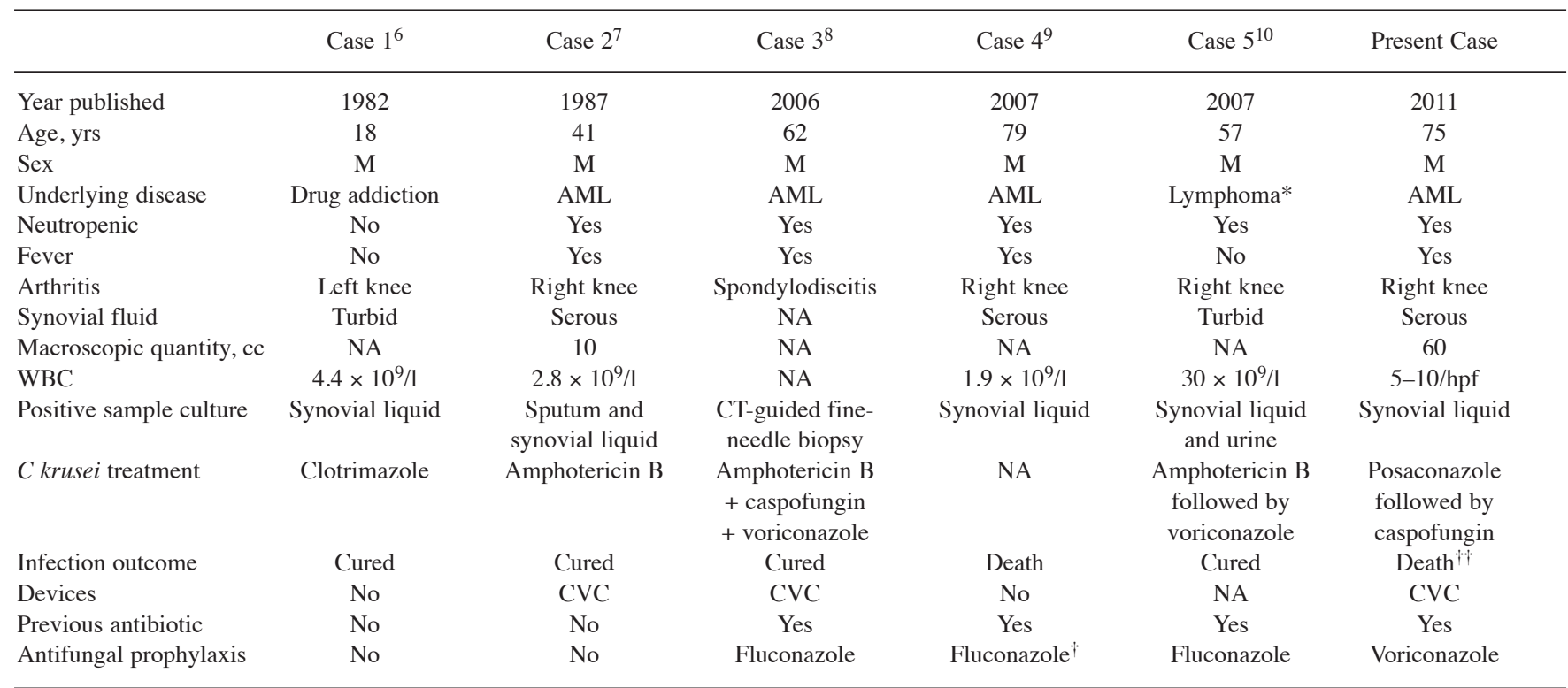

AML: acute myelogenous leukemia; WBC: white blood cell count; NA: not available; CVC: central venous catheter. * Diffuse B cell lymphoma. ${ }^{\dagger}$ Patient was receiving treatment with fluconazole because of $C$. tropicalis arthritis. $C$. krusei was isolated in subsequent arthrocentesis. ${ }^{\dagger \dagger}$ Bacterial infection. 
as the port of entry. It is likely that he became infected with this organism at this time, before he had achieved full, steady, effective levels of voriconazole. Once $C$. krusei colonized the joint, it may have been more difficult to achieve the levels needed to suppress the infection in the synovial fluid. A case report of voriconazole levels in synovial fluid in humans showed them to be lower than serum levels ${ }^{11}$. In addition, with the lack of neutrophils, the host was unable to clear the fungal infection.

In general, in a patient with intact host immunity, most septic arthritis is bacterial and is generally treated with appropriate antibiotics alone. The therapeutic responses are excellent if the infection is diagnosed early. Our patient, however, was neutropenic and immunocompromised, and had prior antibacterial and antifungal prophylaxis, all risk factors for opportunistic infections. Empiric therapy in susceptible hosts presenting with acute monoarthritis should include not only antibacterial, but also antifungal agents effective for emerging non- $C$. albican species like the one isolated in our case. Finally, a delay in clinical response may require switching or adding therapies, particularly if there is uncertainty about the levels attained by specific antiinfective agents in the joint.

HUIFANG LU, MD, PhD; MARIA F. MARENGO, MD, Section of Rheumatology, Department of General Internal Medicine; CORALIA N. MIHU, MD, Department of Infectious Disease; GUILLERMO GARCIA-MANERO, MD, Department of Leukemia; MARIA E. SUAREZ-ALMAZOR, MD, PhD, Section of Rheumatology, Department of General Internal Medicine, The University of Texas M.D. Anderson Cancer Center, Houston, Texas, USA.

Address correspondence to Dr. H. Lu, Department of General Internal Medicine, The University of Texas M.D. Anderson Cancer Center, 1515 Holcombe Blvd., Unit 1465, Houston, Texas, USA.

E-mail: hlu@mdanderson.org

\section{REFERENCES}

1. Cuellar ML, Silveira LH, Espinoza LR. Fungal arthritis. Ann Rheum Dis 1992;51:690-7.

2. Leventakos K, Lewis RE, Kontoyiannis DP. Fungal infections in leukemia patients: How do we prevent and treat them? Clin Infect Dis 2010;50:405-15.

3. Krcmery V Jr, Spanik S, Kunova A, Trupl J, Grausova S, Krupova I, et al. Nosocomial Candida krusei fungemia in cancer patients: Report of 10 cases and review. J Chemother 1999;11:131-6.

4. Samaranayake YH, Samaranayake LP. Candida krusei: Biology, epidemiology, pathogenicity and clinical manifestations of an emerging pathogen. J Med Microbiol 1994;41:295-310.

5. Krcmery V, Barnes AJ. Non-albicans Candida spp. causing fungaemia: Pathogenicity and antifungal resistance. J Hosp Infect 2002;50:243-60.

6. Carcassi A, Saletti M, Boschi S. [Acute Candida arthritis. Isolation of Candida krusei in a heroin addict]. Minerva Med 1982; 73:2905-9.

7. Nguyen VQ, Penn RL. Candida krusei infectious arthritis. A rare complication of neutropenia. Am J Med 1987;83:963-5.

8. Peman J, Jarque I, Bosch M, Canton E, Salavert M, de Llanos R, et al. Spondylodiscitis caused by Candida krusei: Case report and susceptibility patterns. J Clin Microbiol 2006;44:1912-4.

9. Wang HP, Yen YF, Chen WS, Chou YL, Tsai CY, Chang HN, et al. An unusual case of Candida tropicalis and Candida krusei arthritis in a patient with acute myelogenous leukemia before chemotherapy. Clin Rheumatol 2007;26:1195-7.

10. Sili U, Yilmaz M, Ferhanoglu B, Mert A. Candida krusei arthritis in a patient with hematologic malignancy: Successful treatment with voriconazole. Clin Infect Dis 2007;45:897-8.

11. Denes E, Boumedience A, Durox H, Oksman A, Saint-Marcoux F, Darde ML, et al. Voriconazole concentrations in synovial fluid and bone tissues. J Antimicrob Chemother 2007;59:818-9.

J Rheumatol 2012;39:6; doi:10.3899/jrheum.111348 